\title{
Identification and Validation of Expressed Sequence Tags from Pigeonpea (Cajanus cajan L.) Root
}

\author{
Ravi Ranjan Kumar, ${ }^{1,2}$ Shailesh Yadav, ${ }^{3}$ Shourabh Joshi, ${ }^{3}$ Prithviraj P. Bhandare, ${ }^{2}$ \\ Vinod Kumar Patil, ${ }^{2}$ Pramod B. Kulkarni, ${ }^{2}$ Swati Sonkawade, ${ }^{2}$ and G. R. Naik ${ }^{2}$ \\ ${ }^{1}$ Vidya Pratishthan's School of Biotechnology, Vidyanagari, Baramati, Pune 413133, India \\ ${ }^{2}$ Department of Biotechnology, Gulbarga University, Gulbarga, Karnataka 585106, India \\ ${ }^{3}$ Institute of Biotechnology, Acharya N. G. Ranga Agricultural University, Hyderabad 500030, India \\ Correspondence should be addressed to G. R. Naik; grnaikbiotech@gmail.com
}

Received 14 November 2013; Accepted 15 April 2014; Published 6 May 2014

Academic Editor: Søren K. Rasmussen

Copyright (C) 2014 Ravi Ranjan Kumar et al. This is an open access article distributed under the Creative Commons Attribution License, which permits unrestricted use, distribution, and reproduction in any medium, provided the original work is properly cited.

Pigeonpea (Cajanus cajan (L) Millsp.) is an important food legume crop of rain fed agriculture in the arid and semiarid tropics of the world. It has deep and extensive root system which serves a number of important physiological and metabolic functions in plant development and growth. In order to identify genes associated with pigeonpea root, ESTs were generated from the root tissues of pigeonpea (GRG-295 genotype) by normalized cDNA library. A total of 105 high quality ESTs were generated by sequencing of 250 random clones which resulted in 72 unigenes comprising 25 contigs and 47 singlets. The ESTs were assigned to 9 functional categories on the basis of their putative function. In order to validate the possible expression of transcripts, four genes, namely, S-adenosylmethionine synthetase, phosphoglycerate kinase, serine carboxypeptidase, and methionine aminopeptidase, were further analyzed by reverse transcriptase PCR. The possible role of the identified transcripts and their functions associated with root will also be a valuable resource for the functional genomics study in legume crop.

\section{Introduction}

Pigeonpea (Cajanus cajan L.) Millsp. $(2 n=22)$ is a major grain legume of the arid and semiarid regions of the world [1]. Though considered a minor crop, pigeonpea is of considerable importance in areas of South Asia (mainly on the Indian subcontinent), Africa, the Caribbean, and Latin America, where it is a prominent source of protein in the human diet, as well as wood for fuel and light duty structural applications such as thatch for roofing [2]. Pigeonpea has now moved from an "orphan legume crop" to one of the promising pluses where genomics-assisted breeding approaches for a sustainable crop improvement are routine by Pigeonpea Genome Initiative, an effort of various researchers [3]. The first pigeonpea EST dataset provides a transcriptomic resource for gene discovery and development of functional markers associated with biotic stress resistance [4]. Root is the major part of water and nutrition uptake in pigeonpea which has a deep and extensive root system that provides access to water stored deep in the soil profile when that in the surface layer is depleted; this source of water is particularly important for long duration crops. In order to identify the associated genes in pigeonpea root tissues, a normalized cDNA library was constructed from pigeonpea root and expression analysis of the identified genes was carried out by reverse transcriptase PCR (RT-PCR) technique.

\section{Materials and Methods}

The pigeonpea genotype, namely, GRG-295 was selected to construct cDNA library and identification of expressed sequence tags (ESTs). The seeds of GRG-295 pigeonpea genotype were grown in petri dish for 15 days and irrigated with water. At the end of the 15th day, total RNA was isolated from root tissues by using the Trizol reagent (Invitrogen, Carlsbad, CA, USA), and mRNA was further isolated by using the PolyATract mRNA Isolation System 
TABle 1: Primer sequences of subset of ESTs for RT-PCR analysis.

\begin{tabular}{|c|c|c|c|}
\hline Accession number & Putative function & Optimum $T_{m}\left({ }^{\circ} \mathrm{C}\right)$ & Primer sequence $\left(5^{\prime}-3^{\prime}\right)$ \\
\hline \multirow{2}{*}{ JK973671 } & \multirow{2}{*}{ S-Adenosylmethionine synthetase } & \multirow[t]{2}{*}{ (2) } & F-AGAGGAAAT CGGT GCTGGTG \\
\hline & & & R-GCAGCAATTTGGTCGTTGGT \\
\hline \multirow{2}{*}{ JK973674 } & \multirow{2}{*}{ Phosphoglycerate kinase } & \multirow{2}{*}{59} & F-TCCCGATCCCGATACCCTAC \\
\hline & & & R-CAGCACGCTTTTCAGCAGTT \\
\hline \multirow{2}{*}{ JK973715 } & \multirow{2}{*}{ Serine carboxypeptidase } & \multirow{2}{*}{60} & F-ACATGAAGCTCAGTGGAGGAG \\
\hline & & & R-AGCCATGGCCTCCAATCTTC \\
\hline \multirow{2}{*}{ JK973726 } & \multirow{2}{*}{ Methionine aminopeptidase } & \multirow{2}{*}{60} & F-GGCAT TGAAAGTTGGGCAGG \\
\hline & & & R-GATTGCAGCACCGACATCAC \\
\hline
\end{tabular}

(Promega, Madison, WI, USA). The quantification of RNA was verified by absorption ratio of $\mathrm{OD}_{260 / 280}$ and by formaldehyde gel electrophoresis. The first and second strands of cDNA were synthesized using Clontech SMARTer PCR cDNA Synthesis Kit. The cDNAs were purified by the MinElute PCR purification kit (Qiagen, Valencia, CA, USA) and ligated into a pGEM-T easy vector (Promega, Madison, WI, USA). Ligated plasmid DNAs were used for transformation into competent E. coli $\mathrm{DH} 5 \alpha$ strain. Positive clones were selected on an ampicillin/IPTG/X-Gal LB plate. Plasmid DNA from positive clones were isolated by using REAL 96 plasmid isolation kit (Qiagen, Netherlands), and purified DNA was used for single-pass Sanger sequencing by using $\mathrm{M} 13 \mathrm{~F} / \mathrm{R}$ universal sequencing primers on $\mathrm{ABI}$ sequencing machine 3500XL Genetic analyzer. All the ESTs were processed using VecScreen (http://www.ncbi.nlm.nih .gov/VecScreen/VecScreen.html) to remove vector and cloning oligo sequences and various contaminants to trim a high quality region. Based on the qualified sequences, the predicted amino acid sequences were used to search for similar peptide sequences to search for similar protein sequences in public database NCBI (http://www.ncbi.nlm.nih.gov) using the BLASTx search algorithm [5] by using default parameters of the program. The similarity scores between the cDNA clones and known sequences were represented by BLASTx probability $E$ values. Further the ESTs were classified into different functional categories based on the knowledge of biochemistry, plant physiology, and molecular biology (http://www.MetaCyc.org/), GO (http://www.ebi.ac.uk/), and COG (http://www.ncbi.nlm.nih.gov/COG/) tools and by searching related abstracts in PubMed.

2.1. RT-PCR Analysis. Total root RNAs isolated from pigeonpea root tissues were used for reverse transcription polymerase chain reaction (RT-PCR) analysis. Genomic DNA contamination was removed by DNase I. First-strand cDNA was synthesized from each $2 \mu \mathrm{g}$ of total RNA sample using Clontech SMARTer PCR cDNA Synthesis Kit according to the manufacturer's protocol. The cDNAs were purified using a commercial column (Qiagen). To determine the expression of candidate genes, PCR was performed with $2 \mu \mathrm{L}$ of the first-strand CDNA template and gene-specific primer pairs. Gene-specific RT-PCR primers were designed with Primer 3.0 according to the EST sequences and were synthesized commercially. General PCR was conducted with annealing as required for the specific primer pairs (Table 1). RT-PCR experiments were repeated three times, and the PCR products were detected on $1.5 \%$ agarose gel.

\section{Results and Discussion}

Plant root systems serve a number of important functions, including anchoring the plant, absorbing water and nutrients, producing amino acids and hormones, and secreting organic acids, enzymes, and alkaloids [6]. The physiological significance of roots is belied by their relative structural simplicity as compared to other plant organs; major metabolic pathways such as photosynthesis lacking in root tissues have a stereotypical morphology that is conserved across taxa and throughout the life cycle of individuals. This combination of physiological relevance and structural simplicity has made roots obvious targets for functional genomics analyses [7]. As a major grain legume of semiarid tropics and a deep and extensive root system of pigeonpea represents an excellent source of identification of ESTs associated with their root tissues. So, the present work was focused on the study of genes associated with pigeonpea root tissues.

In the present investigation, the cDNA library was constructed in order to identify ESTs associated with pigeonpea roots and their functional analysis was carried out. The total RNA from the pigeonpea root tissue was isolated and the first and second cDNA strand was synthesized. The presence of the gene in plasmid construct of colonies was confirmed by colony PCR. The colony PCR showed that the size of these inserts ranged from 200 to $800 \mathrm{bp}$. Out of 400 bacterial clones, the plasmid construct of 250 positive recombinant clones was sequenced in single passed sequencing reaction from $3^{\prime}$ end using M13 forward/reverse primer and the sequence data was subjected to BLAST analysis. The leading sequences, tailing of the sequence, and poor quality sequences were excluded firstly. Finally, 105 high quality ESTs were retained which were clustered into 72 unigenes comprising 25 contigs and 47 singlets (Table 2) and were compared with NCBI nonredundant protein database using BLASTx algorithm and default parameters. In BLASTx analysis, it was shown that most of the sequences were having a significant homology with known proteins. Sequences that had no significant homology with protein database were compared to nucleotide BLAST using default parameters. 
TABLE 2: Summary of ESTs library.

\begin{tabular}{lc}
\hline Total clone sequenced & 250 \\
ESTs taken for analysis & 105 \\
Number of unigenes & 72 \\
Number of contigs & 25 \\
Number of singlets & 47 \\
Average length of unigenes & $442 \mathrm{bp}$ \\
Average length of ESTs & $437 \mathrm{bp}$ \\
\% GC content of unigenes & 50.3 \\
\% GC content of ESTs & 51.2 \\
\hline
\end{tabular}

The ESTs were deposited to NCBI dbEST with the accession number of JK973637 to JK973741 (Table 3).

The ESTs were categorized into 8 diverse functional group consisting of $10 \%$ transporter genes, $6 \%$ signal transduction genes, $20 \%$ cell growth and transcriptional regulator genes, and $26 \%$ metabolism genes. In other genes, $8 \%$ genes were uncharacterized, $8 \%$ hypothetical genes, $10 \%$ genes with no significant match, and $14 \%$ genes was involved in other functions. There were $8 \%$ of the ESTs found that did not show any match to known proteins in BLASTx program and that suggest novel nature of those genes (Figure 1).

In order to validate the ESTs generated from cDNA library, the expression of the four genes which were involved in different metabolic pathways was analysed by RT-PCR. RT-PCR results showed that the expression levels of four candidate genes, namely; S-adenosylmethionine synthetase, phosphoglycerate kinase, serine carboxypeptidase, and methionine aminopeptidase, were clearly expressed in pigeonpea roots (Figure 2). It was concluded that overall, there was a good agreement between the cDNA library data and the RTPCR results.

S-Adenosylmethionine synthetase (SAMS) comprises of two cDNAs in Pinus contorta among which SAMS1 is expressed in roots and exhibits a specific expression pattern in the meristem at the onset of adventitious root development [8]. SAMS also catalyses the nucleophilic substitution reaction from between methionine and ATP into S-adenosylmethionine which have central role in several biological process in plants, namely, methyl group donor in trimethylation of lignin, DNA, and alkaloids as well as donor of aminopropyl moieties in ethylene and polyamine synthesis $[8,9]$.

Phosphoglycerate kinase superfamily has a diverse function in numerous metabolic processes like generation of precursor metabolites and energy, carbohydrate metabolism, phosphorous metabolism, glycolysis, kinase activity, ATP binding activity, and so forth. The presence of phosphoglycerate kinase transcripts in the cDNA library supports its diverse function in pigeonpea root.

Serine carboxypeptidase differentially expressed in root and other tissues is responsible for the synthesis of sinapoylcholine and sinapoylmalate in Arabidopsis which encodes 51 proteins annotated as serine carboxypeptidase-like enzymes and emerged as a new group of acyltransferase enzymes that are able to modify plant natural products [10-12].

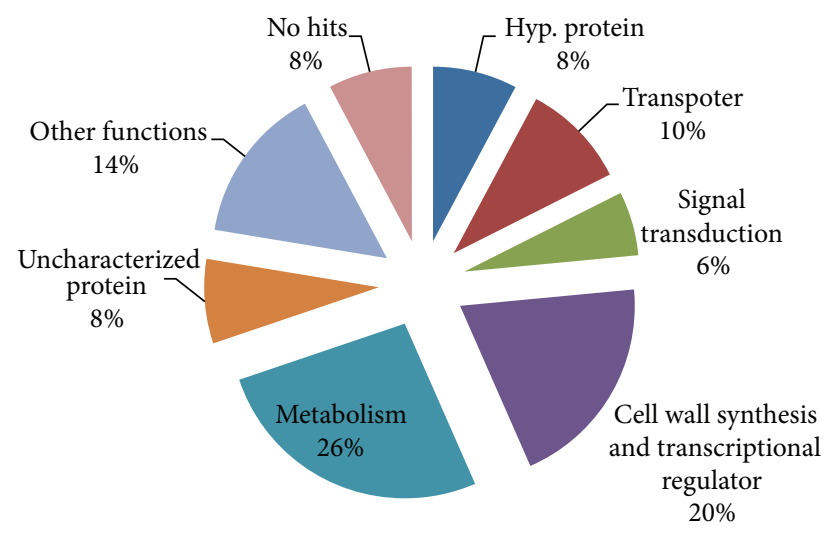

FIGURE 1: Functional classification of ESTs of pigeonpea generated by single pass sequencing.

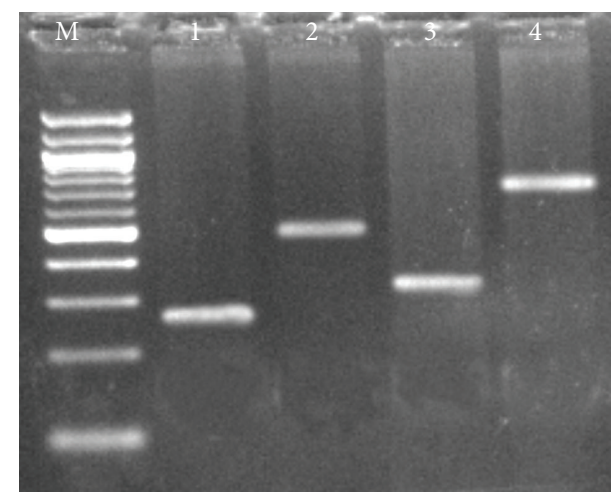

FIGURE 2: Validation of cDNA library results by RT-PCR (M100 bp ladder, 1-S-adenosylmethionine synthetase; 2-phosphoglycerate kinase; 3-serine carboxypeptidase; and 4-methionine aminopeptidase).

Methionine aminopeptidase, a ubiquitous enzyme, differentially expressed in root tissues is one of the central enzymes in protein synthesis that catalyzes $\mathrm{N}$-terminal methionine from proteins [13].

A few important ESTs, viz., type 2 metallothionein, TBPassociated factor, $\mathrm{ABC}$ transporter, and phosphatidylcholine transfer protein, were also abundantly found in the cDNA library. Reddy et al. [14] found abundant metallothionein genes in normalized library from rice leaves and suggested that they might perform essential functions of plant growth beside metal detoxification. TATA-binding protein and TBPassociated factors are transcriptional factors which are predominantly involved in RNA polymerase II mediated transcription process [15]. ABC transporters play an important role in organ growth, plant nutrition, plant development, response to abiotic stress, and the interaction of the plant with its environment [16]. Phosphatidylcholine is usually the most abundant phospholipids in animals and plants, often amounting to almost $50 \%$ of the total, and as such it is obviously the key building block of membrane bilayers making up a very high proportion of the outer leaflet of 


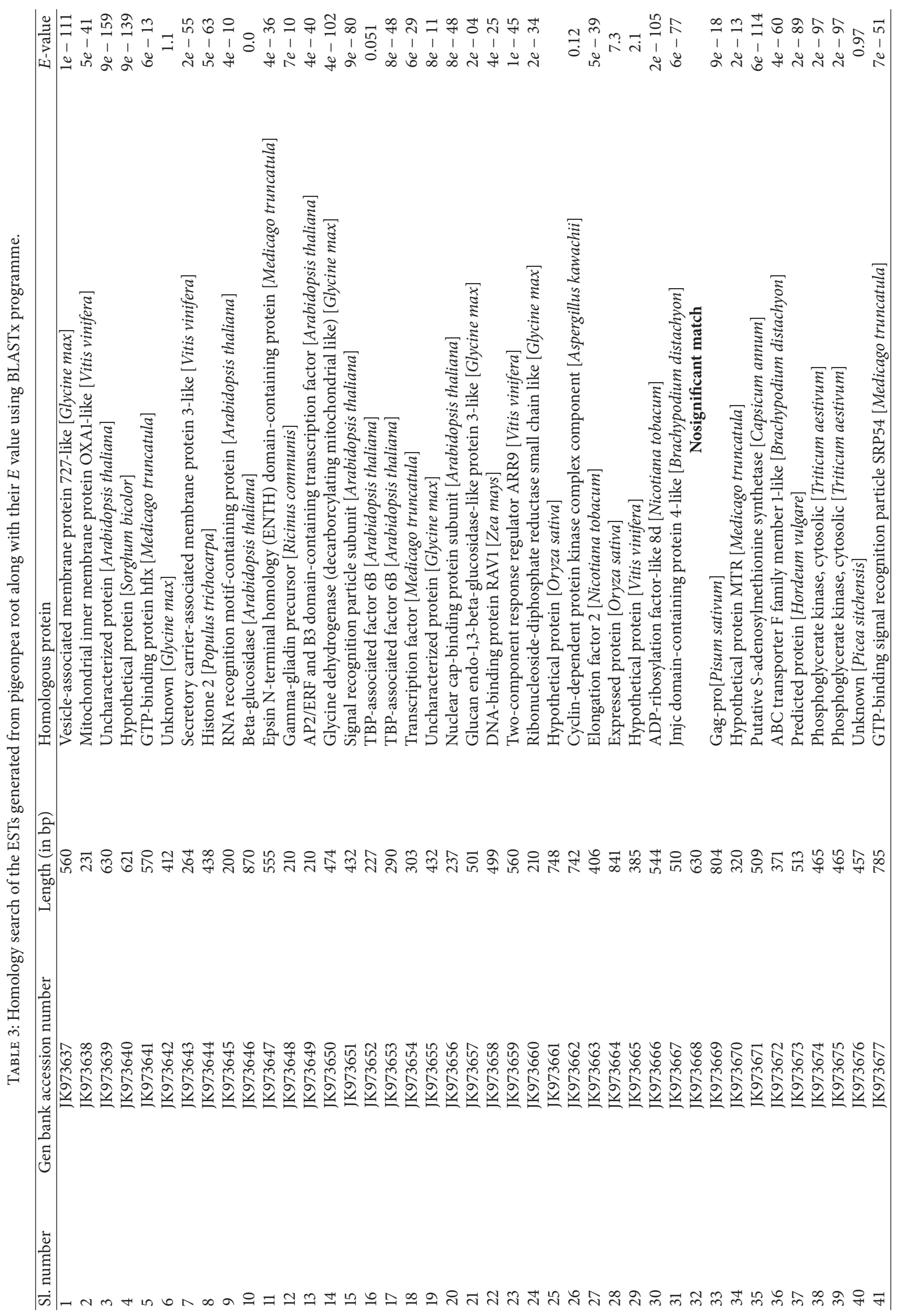




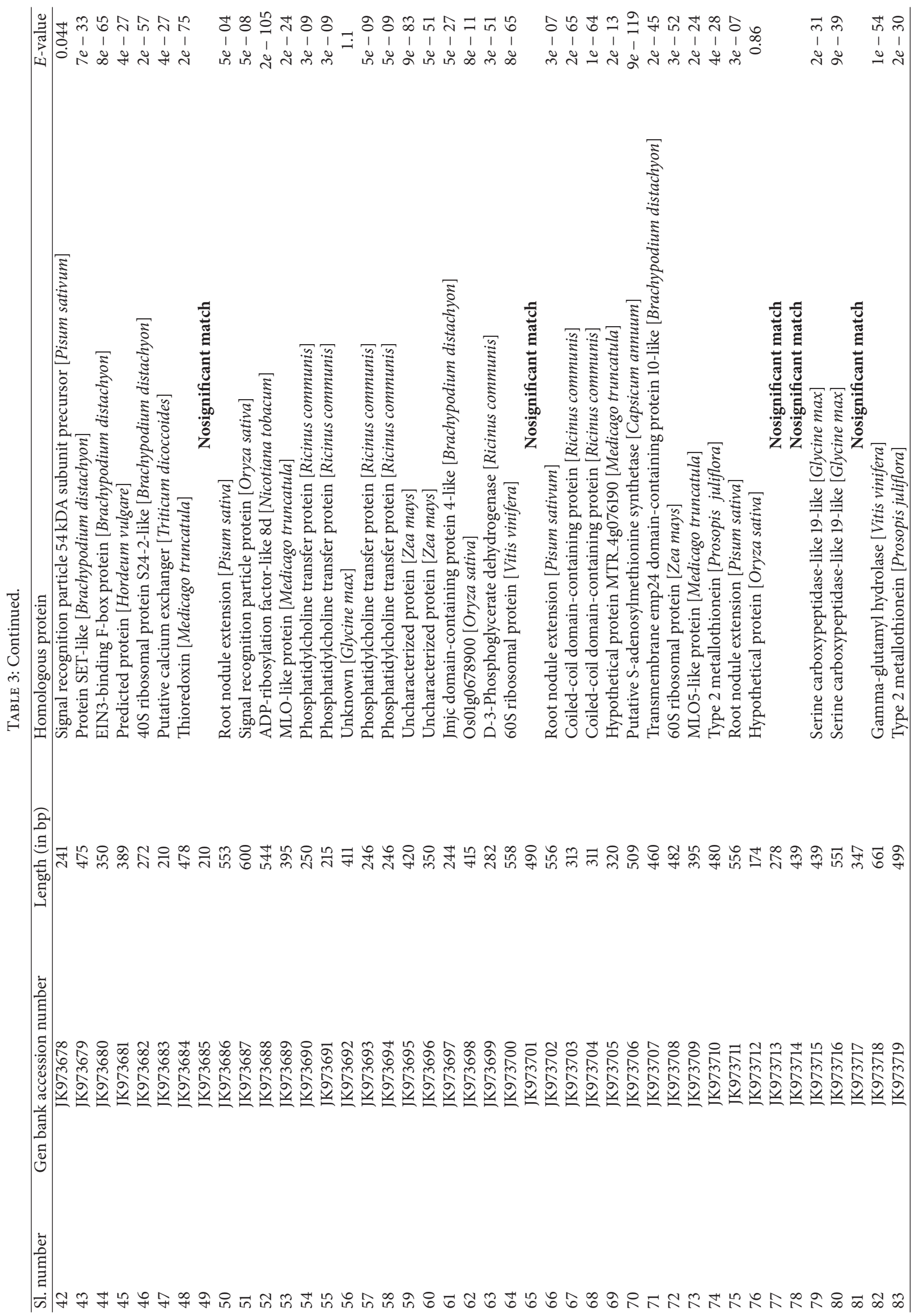




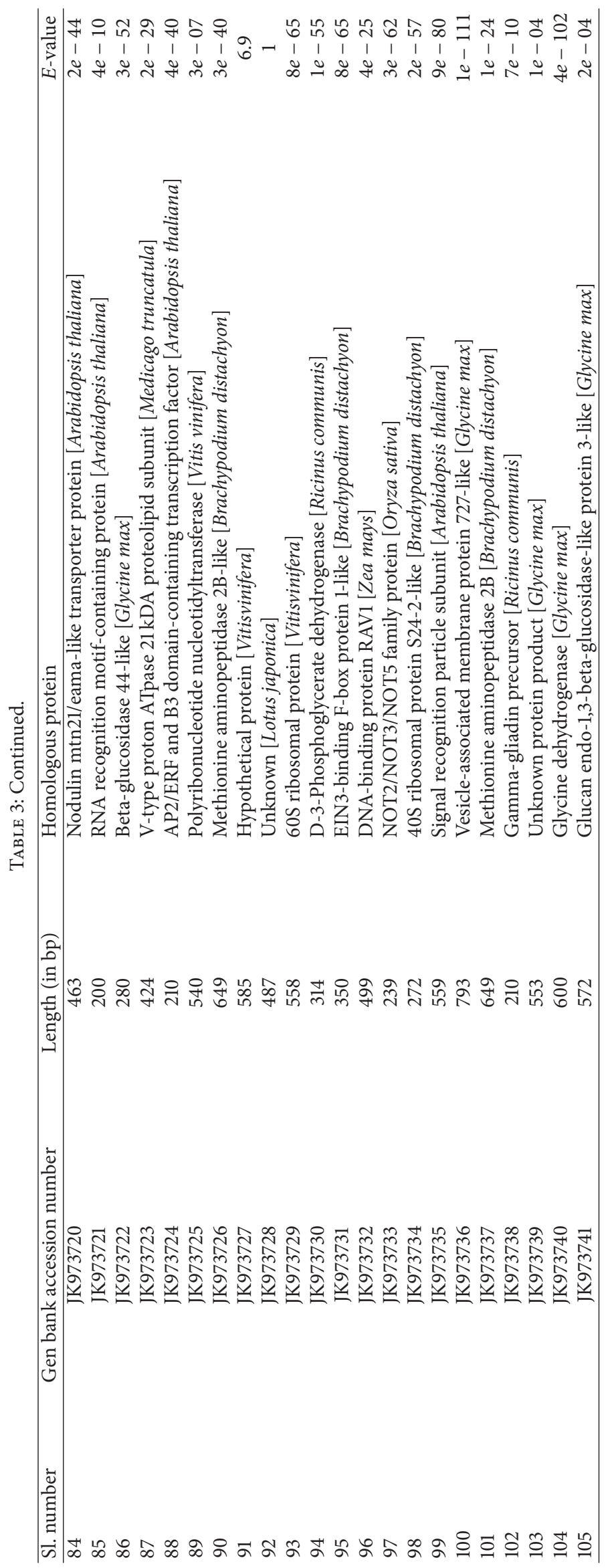


the plasma membrane [17]. Similarly numerous ESTs like glycine dehydrogenase, signal proteins, root nodule extensions, membrane proteins, and $\beta$-glucosidase were observed in the cDNA library constructed from pigeonpea root tissues, which is thought to play possible roles in plant metabolism, growth, and development.

Apart from the known ESTs, 6 EST transcripts (JK973668, JK973685, JK973701, JK973714, JK973715, and JK973718) were observed as not having any significant match in NCBI database. Along with that uncharacterized and hypothetical proteins were also observed in cDNA library which were thought to impart in the cardinal role in root tissues of pigeonpea.

\section{Conclusion}

Our present investigation contains a precise repertoire of transcripts associated with the various metabolic functions in pigeonpea root. These ESTs appear to be involved in multiple metabolism pathways in the plant's physiological and biochemical processes. In addition to known genes, some ESTs were unknown and uncharacterized, whose functional roles remain unclear and require further investigation in future. The root transcriptome characterized in this study markedly provides a unique resource for investigating the functional specificities of the root system. These EST tags may be useful for functional gene annotation, analysis of splice site variants and intron/exon determination, and evaluation of gene homologies or KEGG pathway confirmation.

\section{Abbreviations}

ESTs: $\quad$ Expressed sequence tags

RT PCR: Reverse transcription polymerase chain reaction

SAMS: S-Adenosyl methionine synthetase.

\section{Conflict of Interests}

The authors declare that there is no conflict of interests regarding the publication of this paper.

\section{References}

[1] Y. L. Nene and V. K. Shiela, "Pigeon pea: geography and importance," in The Pigeon Pea, Y. L. Nene, S. H. Hall, and V. K. Sheila, Eds., pp. 1-14, CAB International, Wellingford, UK, 1990.

[2] M. T. Kassa, R. V. Penmetsa, N. Carrasquilla-Garcia et al., "Genetic patterns of domestication in pigeonpea (Cajanus cajan (L.) Millsp.) and wild Cajanus relatives," PLOS ONE, vol. 7, no. 6, Article ID e39563, 2012.

[3] R. K. Varshney, R. V. Penmetsa, S. Dutta et al., "Pigeonpea genomics initiative (PGI): an international effort to improve crop productivity of pigeonpea (Cajanus cajan L.)," Molecular Breeding, vol. 26, no. 3, pp. 393-408, 2010.

[4] N. L. Raju, B. N. Gnanesh, P. Lekha et al., “The first set of EST resource for gene discovery and marker development in pigeonpea (Cajanus cajan L.)," BMC Plant Biology, vol. 10, pp. 45-51, 2010.
[5] S. F. Altschul, T. L. Madden, A. A. Schäffer et al., "Gapped BLAST and PSI-BLAST: a new generation of protein database search programs," Nucleic Acids Research, vol. 25, no. 17, pp. 3389-3402, 1997.

[6] R. Zhai, Y. Feng, H. Wang et al., "Transcriptome analysis of rice root heterosis by RNA-Seq," BMC Genomics, vol. 14, article 19, pp. 1-14, 2013.

[7] Y. Jiang and M. K. Deyholos, "Comprehensive transcriptional profiling of $\mathrm{NaCl}$-stressed Arabidopsis roots reveals novel classes of responsive genes," BMC Plant Biology, vol. 6, article $25,2006$.

[8] A. M. Lindroth, P. Saarikoski, G. Flygh et al., "Two S-adenosylmethionine synthetase-encoding genes differentially expressed during adventitious root development in Pinus contorta," Plant Molecular Biology, vol. 46, no. 3, pp. 335-346, 2001.

[9] P. K. Chiang, R. K. Gordon, J. Tal et al., "S-adenosylmethionine and methylation," The FASEB Journal, vol. 10, no. 4, pp. 471-480, 1996.

[10] A. M. Shirley, C. M. McMichael, and C. Chapple, "The sng2 mutant of Arabidopsis is defective in the gene encoding the serine carboxypeptidase-like protein sinapoylglucose:choline sinapoyltransferase," Plant Journal, vol. 28, no. 1, pp. 83-94, 2001.

[11] C. M. Fraser, M. G. Thompson, A. M. Shirley et al., "Related Arabidopsis serine carboxypeptidase-like sinapoylglucose acyltransferases display distinct but overlapping substrate specificities," Plant Physiology, vol. 144, no. 4, pp. 1986-1999, 2007.

[12] D. Weier, J. Mittasch, D. Strack, and C. Milkowski, “The genes BnSCT1 and BnSCT2 from Brassica napus encoding the final enzyme of sinapine biosynthesis: molecular characterization and suppression," Planta, vol. 227, no. 2, pp. 375-385, 2008.

[13] W. T. Lowther and B. W. Matthews, "Structure and function of the methionine aminopeptidases," Biochimica et Biophysica Acta, vol. 1477, no. 1-2, pp. 157-167, 2000.

[14] V. S. Reddy, G. S. Ali, and A. S. N. Reddy, "Genes encoding calmodulin-binding proteins in the Arabidopsis genome," Journal of Biological Chemistry, vol. 277, no. 12, pp. 9840-9852, 2002.

[15] S. R. Albright and R. Tjian, "TAFs revisited: more data reveal new twists and confirm old ideas," Gene, vol. 242, no. 1-2, pp. $1-13,2000$.

[16] J. Kang, J. Park, H. Choi et al., "Plant $\mathrm{ABC}$ transporters," The Arabidopsis Book, vol. 9, pp. 1-25, 2010.

[17] K. A. Devor and J. B. Mudd, "Structural analysis of phosphatidylcholine of plant tissue," Journal of Lipid Research, vol. 12, no. 4, pp. 396-402, 1971. 

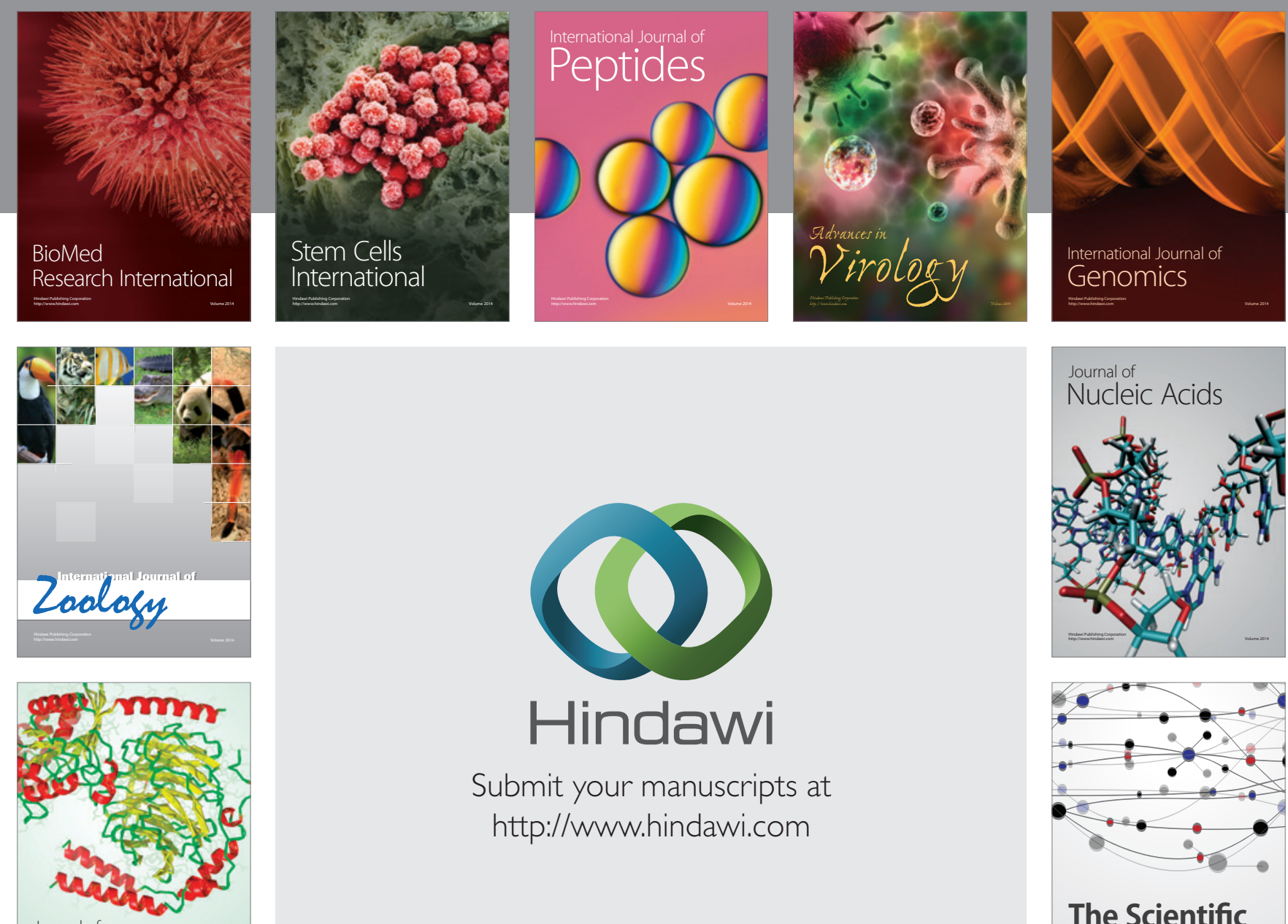

Submit your manuscripts at

http://www.hindawi.com

Journal of
Signal Transduction
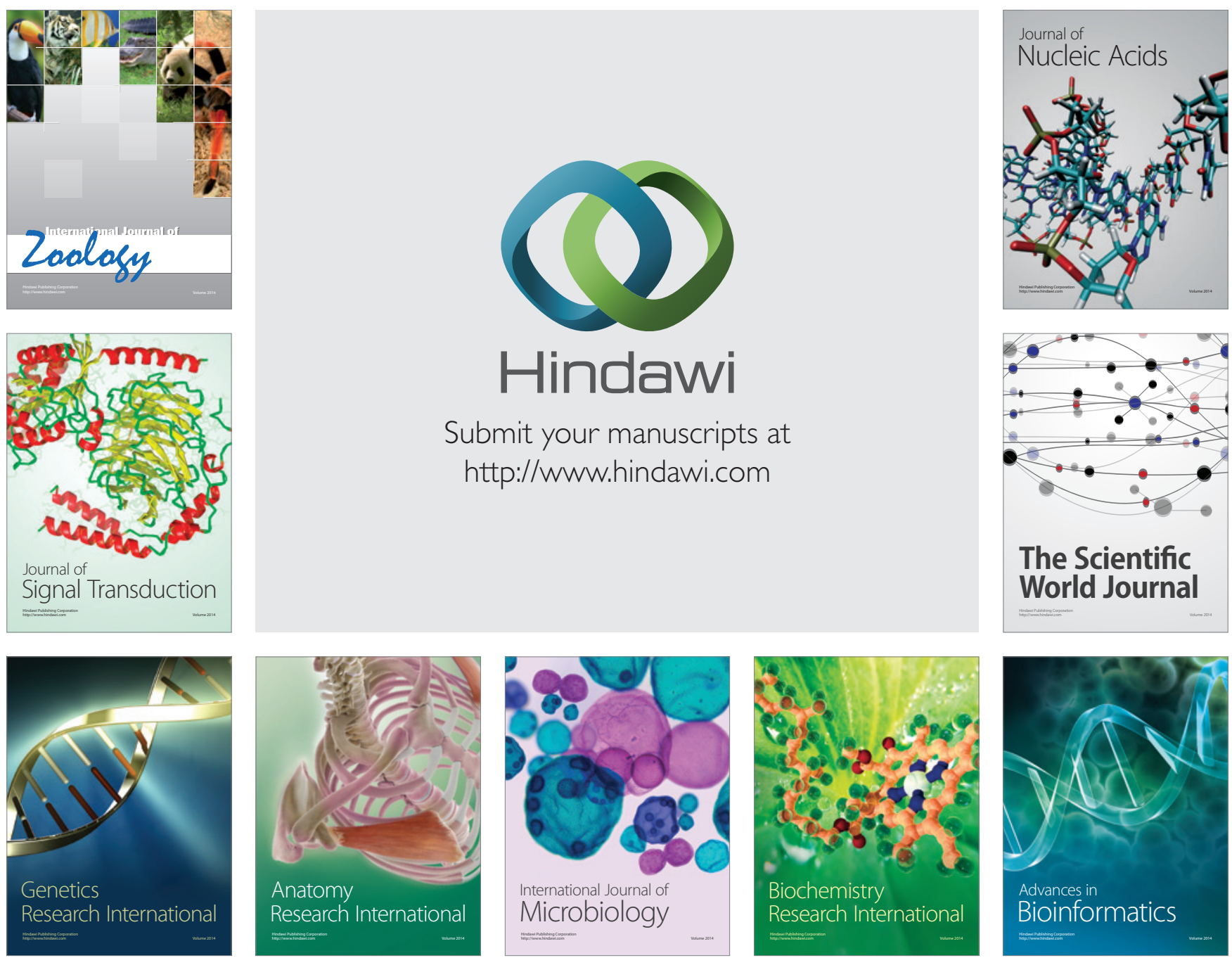

The Scientific World Journal
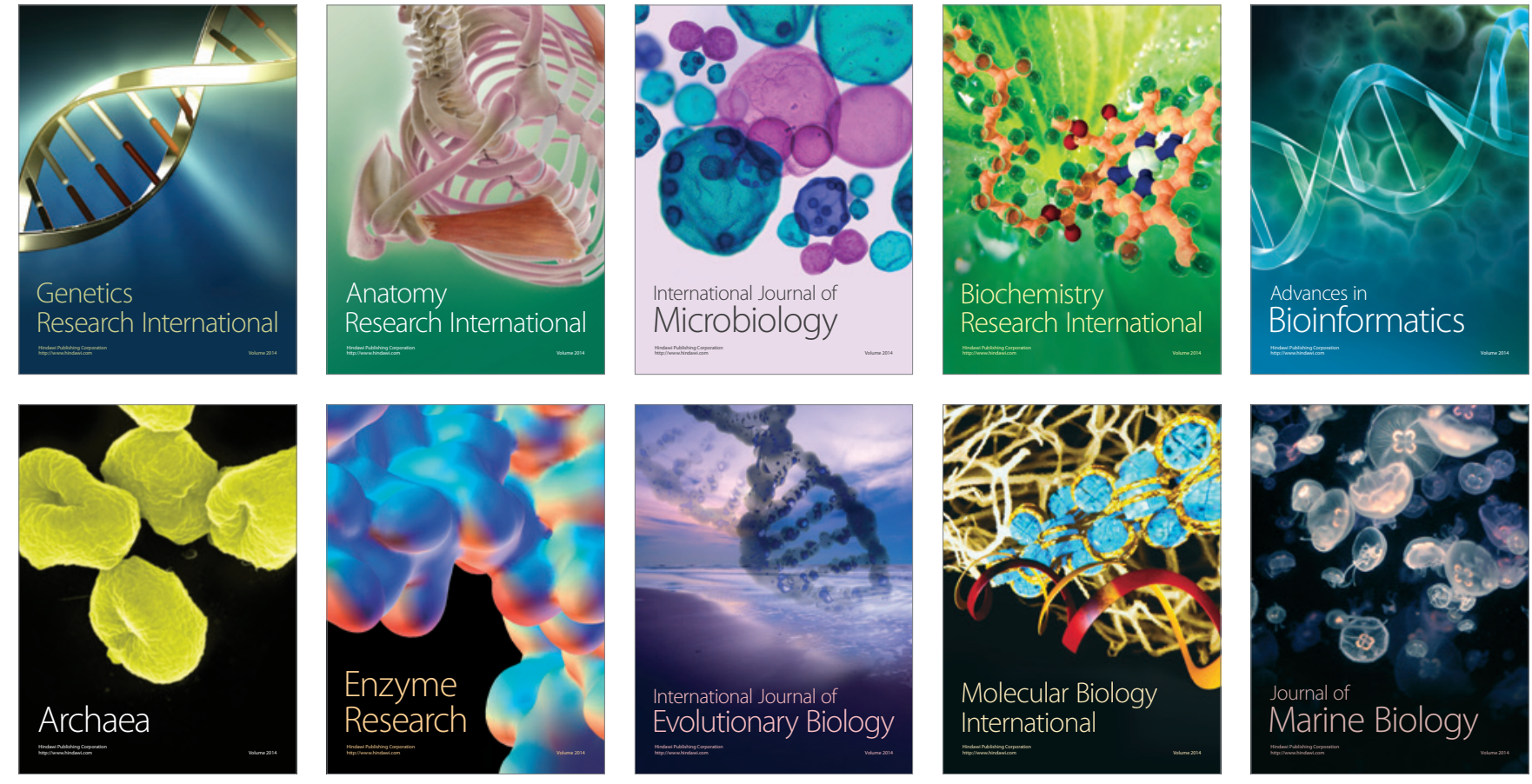